Article

\title{
Gene Cloning, Expression and Characterization of a Novel Xylanase from the Marine Bacterium, Glaciecola mesophila KMM241
}

\author{
Bing Guo ${ }^{\dagger}$, Ping-Yi Li ${ }^{\dagger}$, Yong-Sheng Yue, Hui-Lin Zhao, Sheng Dong, Xiao-Yan Song, \\ Cai-Yun Sun, Wei-Xin Zhang, Xiu-Lan Chen, Xi-Ying Zhang *, Bai-Cheng Zhou and \\ Yu-Zhong Zhang
}

The State Key Lab of Microbial Technology, Marine Biotechnology Research Center, Shandong University, Jinan 250100, China; E-Mails: guo321bing@163.com (B.G.); lipingyipeace@163.com (P.-Y.L.); yueyongsheng1124@163.com (Y.-S.Y.); zhaohuilin1984@yahoo.cn (H.-L.Z.); Ds518@126.com (S.D.); xysong@sdu.edu.cn (X.-Y.S.); suncy@sdu.edu.cn (C.-Y.S.); zhangweixin1984@163.com (W.-X.Z.); cx10423@sdu.edu.cn (X.-L.C.); zhangxiying@sdu.edu.cn (X.-Y.Z.); bczhou@ms.qdio.ac.cn (B.-C.Z.); zhangyz@sdu.edu.cn (Y.-Z.Z.)

$\dagger$ These authors contributed equally to this work.

* Author to whom correspondence should be addressed; E-Mail: zhangxiying@sdu.edu.cn; Tel.: +86-531-88364326; Fax: +86-531-88564326.

Received: 14 January 2013; in revised form: 6 March 2013 / Accepted: 19 March 2013 / Published: 8 April 2013

\begin{abstract}
Marine xylanases are rather less studied compared to terrestrial xylanases. In this study, a new xylanase gene, $x y n B$, was cloned from the marine bacterium, Glaciecola mesophila KMM241, and expressed in Escherichia coli. xynB encodes a multi-domain xylanase XynB of glycoside hydrolase $(\mathrm{GH})$ family 8 . The recombinant XynB comprises an $N$-terminal domain (NTD) with unknown function and a catalytic domain, which is structurally novel among the characterized xylanases of GH family 8 . $\mathrm{XynB}$ has the highest identity (38\%) to rXyn8 among the characterized xylanases. The recombinant XynB showed maximal activity at $\mathrm{pH} 6-7$ and $35^{\circ} \mathrm{C}$. It is thermolabile and salt-tolerant. $\mathrm{XynB}$ is an endo-xylanase that demands at least five sugar moieties for effective cleavage and to hydrolyze xylohexaose and xylopentaose into xylotetraose, xylotriose and xylobiose. NTD was expressed in Escherichia coli to analyze its function. The recombinant NTD exhibited a high binding ability to insoluble xylan and avicel and little binding ability to chitosan and chitin. Since the NTD shows no obvious homology to
\end{abstract}


any known carbohydrate-binding module (CBM) sequence in public databases, XynB may contain a new type of CBM.

Keywords: xylanase; XynB; cold-active; Glaciecola mesophila KMM241; carbohydrate-binding module

\section{Introduction}

Xylan, which together with lignin and cellulose constitutes the major components of plant cell walls, is the most abundant hemicellulose in nature and represents an important type of renewable resource [1]. Complete biodegradation of xylan requires synergy of a variety of xylanolytic enzymes. According to the amino acid sequences of catalytic domains, enzymes with xylanase activity can be categorized into glycoside hydrolase $(\mathrm{GH})$ families 5, 7, 8, 10, 11, 16, 26, 43, 52 and 62. However, only those sequences classified into families 5, 7, 8, 10, 11 and 43 contain truly distinct catalytic domains with a demonstrated endo-1,4- $\beta$-xylanase activity. Family 16, 52 and 62 enzymes are bifunctional enzymes containing two catalytic domains, a family 10 or 11 xylanase domain and a glycosidase domain. Those enzymes classified into family 26 are endo-1,3- $\beta$-xylanases [2]. Endo- $\beta$-1,4-xylanases (EC 3.2.1.8) are glycosidases, which cleave the internal $\beta$-1,4-xylosidic bonds of the main chain of xylan, producing unbranched or branched xylo-oligosaccharide [3] and, thus, are widely used in food, paper, textile and many other industries [4,5]. Xylanases from GH families 10 and 11 have been widely investigated. In contrast, xylanases from other families are less studied. GH family 8 comprises endoxylanases, as well as xylose-releasing exo-oligoxylanases (EC 3.2.1.156) [6]. Until now, only four GH family 8 endo-xylanases have been identified, including recombinant xylanase XynY from Bacillus sp. KK-1 [7], xylanase PhXyl from Pseudoalteromonas haloplanktis [8], xylanase XYL6806 from an insect gut microbe [9] and xylanase rXyn8 from an uncultured bacterium [10]. In addition, two GH family 8 exo-xylanases have been reported, one from Bacillus halodurans [11] and the other from Bifidobacterium adolescentis [12].

At present, there is an increasing interest in searching for new sources of xylanases, especially those with extremophilic properties, as these enzymes may have an advantage when applied at extreme conditions [2]. Marine microorganisms have been regarded as a reservoir, not only for novel natural products, but also for valuable genes and enzymes. Several xylanases produced by marine microorganisms from special ecological habitats, such as the deep-sea hydrothermal field [13], the Antarctic marine soil [8] and marine sediment [14], were shown to have special properties, such as hyperthermostability [13], cold adaptation [8,14] and salt-tolerance [14,15]. However, studies on marine xylanases are still rare compared to those on terrestrial xylanases.

Glaciecola mesophila KMM241 was isolated from a specimen of marine invertebrate Halocynthia aurantium [16]. In our previous studies, a cold-adapted and salt-tolerant GH family 10

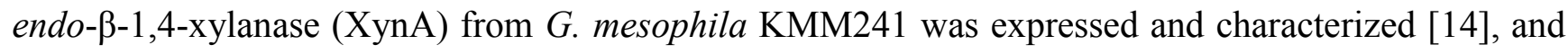
the effect of XynA on bread baking was studied [17]. In this study, another xylanase, XynB, from G. mesophila KMM241 was expressed and characterized, which showed that XynB was a GH family 8 xylanase containing an $N$-terminal domain and a catalytic domain. Moreover, the xylan-binding 
function of the $N$-terminal domain was studied, which suggests that this domain may represent a new type of carbohydrate-binding module (CBM).

\section{Materials and Methods}

\subsection{Strains, Plasmids and Chemicals}

G. mesophila KMM241 was purchased from DSMZ (DSM 15026 ${ }^{\mathrm{T}}$ ). Escherichia coli DH5 $\alpha$ and BL21 were used for gene cloning and expression, respectively. The plasmid, pGME-T (Novagen, Billerica, MA, USA), was used for gene cloning, and the plasmid, pET-22b $(+)$ (Novagen, USA), was used for expression. Beech wood xylan, oat spelt xylan and xylo-oligosaccharides (xylose, xylobiose, xylotriose, xylotetraose, xylopentaose and xylohexaose) were all purchased from Sigma (St louis, MO, USA).

\subsection{Gene Cloning of xynB}

Genomic DNA of G. mesophila KMM241 was prepared with the method described by Saito and Miura [18]. Blast analysis in NCBI showed that the 16S rRNA gene sequence of G. mesophila KMM241 had more than $99 \%$ identity to that of Pseudoalteromonas atlantica T6c. The whole genome of strain T6c was sequenced and released in GenBank, which revealed that there is a gene encoding a hypothetical endo-1,4- $\beta$-xylanase in the genome. Based on the $5^{\prime}$ end and $3^{\prime}$ end sequence of this gene, two primers were designed as follows: XB22-1,5'-CGACGGATCCGGAAGTGAGCATTGATCAC-3'; XB22-2,5'-CGACGCTCGAGTTCAGGCTCGTTTTC-3', in which the cleavage sites of restriction enzymes, BamH I and Xho I, were added and underlined. With these primers and the genomic DNA of strain KMM241 as the template, PCR amplification was performed by Fastpfu polymerase with the following procedure: $95^{\circ} \mathrm{C}$ for $5 \mathrm{~min}, 30$ cycles of $94{ }^{\circ} \mathrm{C}$ for $30 \mathrm{~s}, 50{ }^{\circ} \mathrm{C}$ for $1 \mathrm{~min}$ and $72{ }^{\circ} \mathrm{C}$ for $2 \mathrm{~min}$ and $72{ }^{\circ} \mathrm{C}$ for $10 \mathrm{~min}$. An about $2800 \mathrm{bp}$ DNA fragment was amplified and then sequenced. Sequence analysis showed that it contains a 2739 bp ORF (open reading frame) encoding a hypothetical endo-1,4- $\beta$-xylanase. Then, this gene, named $x y n B$, was deposited in GenBank under accession No. JF514216.

\subsection{Expression and Purification of $X y n B$}

An expression plasmid pET22b- $x y n B$ was constructed by ligating gene $x y n B$ into the BamH I-Xho I restriction sites of the pET22b plasmid. Plasmid pET22b-xynB was introduced into E. coli BL21(DE3) competent cells. The transformed strain was cultured in Luria-Bertani medium supplemented with $0.1 \mathrm{mg} / \mathrm{mL}$ ampicillin overnight at $37^{\circ} \mathrm{C}$. Then, the culture was diluted 100 -fold in fresh Luria-Bertani medium with $0.1 \mathrm{mg} / \mathrm{mL}$ ampicillin and incubated at $37{ }^{\circ} \mathrm{C}$. When the culture reached an optical density of 1.0 at $600 \mathrm{~nm}$, isopropyl- $\beta$-D-thiogalactopyranoside (IPTG, Sigma, USA) was added to a final concentration of $0.2 \mathrm{mM}$, and the culture was further cultivated at $15{ }^{\circ} \mathrm{C}$ for $30 \mathrm{~h}$. Then, the culture was centrifuged at $10,000 \times g$ at $4{ }^{\circ} \mathrm{C}$ for $5 \mathrm{~min}$. The cells were collected, resuspended in $50 \mathrm{mM}$ phosphate buffer ( $\mathrm{pH}$ 7.0) and sonicated. The extract was subjected onto a DEAE-Sepharose Fast Flow column (GE Healthcare, Fairfield, CT, USA) equilibrated with the same buffer. The elution was performed with a linear gradient of $0-0.8 \mathrm{M} \mathrm{NaCl}$. The fraction with xylanase activity was collected and subjected onto a His-Bind metal chelating column (GE Healthcare, USA) equilibrated 
with $50 \mathrm{mM}$ phosphate buffer ( $\mathrm{pH}$ 7.4) containing $500 \mathrm{mM} \mathrm{NaCl}$ and $5 \mathrm{mM}$ imidazole and eluted with $50 \mathrm{mM}$ phosphate buffer ( $\mathrm{pH}$ 7.4) containing $500 \mathrm{mM} \mathrm{NaCl}$ and $150 \mathrm{mM}$ imidazole. The purity of recombinant XynB was detected by SDS-PAGE with the method of Laemmli [19].

\subsection{Enzyme Assay and Protein Determination}

To measure the xylanase activity of $\mathrm{XynB}$, a reaction mixture containing $0.01 \mathrm{~mL}$ enzyme and $0.09 \mathrm{~mL}$ beech wood xylan $(10 \mathrm{mg} / \mathrm{mL})$ dissolved in $50 \mathrm{mM}$ Tris-HCl buffer $(\mathrm{pH} 7.0)$ was incubated at $35{ }^{\circ} \mathrm{C}$ for $10 \mathrm{~min}$. After incubation, $0.15 \mathrm{~mL}$ dinitrosalicylic acid (DNS) was added to end the reaction. The reducing sugar released in the mixture was determined, with xylose as the standard using the DNS method [20]. One unit of enzyme activity was defined as the amount of enzyme capable of releasing $1 \mu \mathrm{mol}$ of xylose per minute under the assay conditions. Protein concentration was estimated with Bradford's method [21].

\subsection{Enzyme Characterization}

To determine the optimal $\mathrm{pH}$ of $\mathrm{XynB}$, xylanase activity was assayed at $35{ }^{\circ} \mathrm{C}$ in different buffers ranging from $\mathrm{pH} 4.0$ to $\mathrm{pH} 11.0$. The buffers used were $50 \mathrm{mM}$ citrate buffer for $\mathrm{pH} 4.0-6.0,50 \mathrm{mM}$ phosphate buffer for $\mathrm{pH} 6.0-8.0,50 \mathrm{mM}$ Tris- $\mathrm{HCl}$ buffer for $\mathrm{pH} 8.0-9.0$ and $50 \mathrm{mM}$ glycine- $\mathrm{NaOH}$ buffer for $\mathrm{pH} 9.0-11.0 \mathrm{pH}$ stability was determined by measuring the residual activity of XynB at $\mathrm{pH} 7.0$ and $35{ }^{\circ} \mathrm{C}$ after the enzyme was incubated in the buffers at $15{ }^{\circ} \mathrm{C}$ for $1 \mathrm{~h}$. The optimal temperature for xylanase activity was determined in $50 \mathrm{mM}$ phosphate buffer $(\mathrm{pH} \mathrm{7.0)}$. The thermostability of $\mathrm{XynB}$ was determined by measuring the residual activity after incubation at $25{ }^{\circ} \mathrm{C}$, $35{ }^{\circ} \mathrm{C}$ and $45{ }^{\circ} \mathrm{C}$ for different periods of time. To analyze the effect of different metal ions and chemical reagents on the activity, 15 kinds of chemicals, including $\mathrm{SnCl}_{2}, \mathrm{CaCl}_{2}, \mathrm{KCl}, \mathrm{SrCl}_{2}, \mathrm{MgCl}_{2}$, $\mathrm{FeCl}_{3}, \mathrm{CuCl}_{2}, \mathrm{LiCl}_{2}, \mathrm{MnCl}_{2}, \mathrm{NiCl}_{2}, \mathrm{ZnCl}_{2}$ and $\mathrm{CoCl}_{2}$, all from Sinopharm Chemical Reagent Co., Ltd. (Shanghai, China), as well as Urea, EDTA and SDS from Sigma (USA), were added to the reaction mixture at final concentrations of $1 \mathrm{mM}$ and $5 \mathrm{mM}$, respectively, and then the xylanase activity was assayed at $\mathrm{pH} 7.0$ and $35^{\circ} \mathrm{C}$. To investigate the effect of $\mathrm{NaCl}$ on the xylanase activity of XynB, 0.1-4.0 $\mathrm{mol} / \mathrm{L} \mathrm{NaCl}$ was added to the reaction mixture, and then, the xylanase activity was assayed at pH 7.0 and $35{ }^{\circ} \mathrm{C}$. Substrate specificity of XynB was tested by measuring its activity toward beech wood xylan, oat spelt xylan, carboxymethylcellulose, laminarin, mannan, starch and chitosan. All reactions were conducted in $50 \mathrm{mM}$ phosphate buffer $(\mathrm{pH} 7.0)$ with a final substrate concentration of $10 \mathrm{mg} / \mathrm{mL}$ at $35{ }^{\circ} \mathrm{C}$ for $10 \mathrm{~min}$. The kinetic parameters of XynB on beech wood xylan and oat spelt xylan were determined at $35^{\circ} \mathrm{C}$ by Lineweaver-Burk plots, which were made by linear regression, with initial rates determined between 1.0 and $15 \mathrm{mg} / \mathrm{mL}$ of substrates.

\subsection{Analysis of the Products of Xylo-Oligosaccharides Hydrolyzed by XynB}

A $50 \mu \mathrm{L}$ reaction solution containing $0.2 \mu \mathrm{g}$ XynB in $50 \mathrm{mM}$ phosphate buffer $(\mathrm{pH} 7.0)$ and $1 \mathrm{mg} / \mathrm{mL}$ xylo-oligosaccharides was incubated at $15{ }^{\circ} \mathrm{C}$ for $12 \mathrm{~h}$. After incubation, the solution was boiled for $5 \mathrm{~min}$ and then centrifuged at $10,000 \times \mathrm{g}$ for $10 \mathrm{~min}$. The supernatant was filtrated through a $0.22 \mu \mathrm{m}$ cellulose acetate membrane for HPLC analysis. The hydrolytic products were analyzed using 
LC-10AD high-performance liquid chromatography (Shimadzu, Japan), equipped with an HPX-42C Aminex column (Bio-Rad, Hercules, CA, USA) and an RID-10A refractive index detector (Shimadzu, Japan). The column was maintained at $75{ }^{\circ} \mathrm{C}$ and eluted with $\mathrm{ddH}_{2} \mathrm{O}$ at a flow rate of $0.4 \mathrm{~mL} / \mathrm{min}$. Xylose and a mixture of xylo-oligosaccharides (x2-x6) were used as the standard.

\subsection{Expression and Purification of the N-Terminal Domain}

A DNA fragment coding for the $N$-terminal domain (NTD) of XynB (Glu44-Arg584) was amplified from $G$. mesophila KMM241 by PCR with the primers, 5'-CGACCATATGGAAGTGAGCATTGA TCAC-3' and 5'-CGACCTCGAGGCGAATATCGTTTGAATC-3', in which the cleavage sites of restriction enzymes, BamH I and Xho I, were added and underlined. An expression plasmid, pET22b- $n t d$, was constructed for the expression of NTD of XynB. pET22b-ntd was transformed into E. coli BL21-(DE3) and induced by $0.5 \mathrm{mM}$ IPTG at $15{ }^{\circ} \mathrm{C}$ for $30 \mathrm{~h}$. The recombinant NTD was expressed as a $C$-terminal $\mathrm{His}_{6}$-tagged protein and purified from the cell extract with a His-Bind metal chelating column. The purity of the recombinant NTD was detected by SDS-PAGE with the methods of Laemmli [19].

\subsection{Insoluble Polysaccharide-Binding Assay of the N-Terminal Domain}

Insoluble xylan was prepared from oat spelt xylan by using the method of Pason et al. [22]. Avicel, chitin and chitosan were purchased from Sigma (USA). The binding ability of the NTD toward these four insoluble polysaccharides was determined by using the method of Valenzuela et al. [23]. The NTD $(0.1 \mathrm{mg})$ was mixed with insoluble polysaccharides $(20 \mathrm{mg})$ in $20 \mathrm{mM}$ sodium phosphate buffer ( $\mathrm{pH} 7.0$ ) in a final volume of $0.2 \mathrm{~mL}$, which was incubated on ice for $1 \mathrm{~h}$ with occasional stirring. After incubation, the mixture was centrifuged at 13,000 rpm for $10 \mathrm{~min}$. The supernatant was carefully removed for SDS-PAGE analysis. Pellets were washed with $0.4 \mathrm{~mL}$ of the same buffer three or four times, and then, the pellets were resuspended in $0.1 \mathrm{~mL}$ of $10 \% \mathrm{SDS}$ and boiled for $10 \mathrm{~min}$ to denature the bound protein. The proteins in the supernatant and the wash buffer of the last time, as well as the released protein from the pellets were analyzed by SDS-PAGE. Bovine serum albumin (BSA, Sigma, USA) instead of the NTD was used as a negative control.

\section{Results}

\subsection{Gene Cloning and Sequence Analysis}

Gene $x y n B$ was cloned from genomic DNA of G. mesophila KMM241. The ORF comprises $2739 \mathrm{bp}$, including an ATG start codon and a TAA stop codon. It encodes a protein of 912 amino acid residues with a calculated $M_{r}$ of $100,502 \mathrm{Da}$, which was predicted to be a GH family 8 xylanase (named XynB). The amino acid sequence of XynB was searched against GenBank databases. Results showed that $\mathrm{XynB}$ is a multi-domain enzyme containing a predicted 43-residue signal peptide at the $N$-terminus (Met1-Ala43), an NTD following the signal peptide (Glu44-Arg584) and a catalytic domain at its $C$-terminus (Ser585-Glu912) (Figure 1). The catalytic domain showed extensive homology with xylanases of GH family 8 [24]. In addition, the multiple sequence alignment (see Figure 2) revealed that XynB contained glutamate and aspartate residues (Glu586, Asp646 and Asp786), which 
are considered to be crucial for catalytic activity of GH family 8 [10]. XynB had high amino acid sequence identities to some hypothetical xylanases in databases, such as the xylanase from Pseudoalteromonas atlantica T6c (98\%, YP660649) and the xylanase from Glaciecola chathamensis S18K6 (80\%, ZP11354175). In contrast, XynB had low identities to characterized xylanases. Among characterized xylanases, XynB had highest identity (38\%) to the xylanase rXyn8 from an uncultured bacterium [10].

Figure 1. Schematic diagrams of the domain structures of $\mathrm{XynB}$ and four other characterized GH family 8 endo-xylanases. XynB, the xylanase in this study (AEC33258); X6806, a xylanase from insect guts (AAS85781) [9]; Xyn8, a cold-active xylanase enzyme from an environmental DNA library (ABB71891) [10]; KK-1, a xylanase from Bacillus sp. KK-1 (AAC27700) [7]; PH, a psychrophilic xylanase from Pseudoalteromonas haloplanktis TAH 3a (AJ427921) [8].

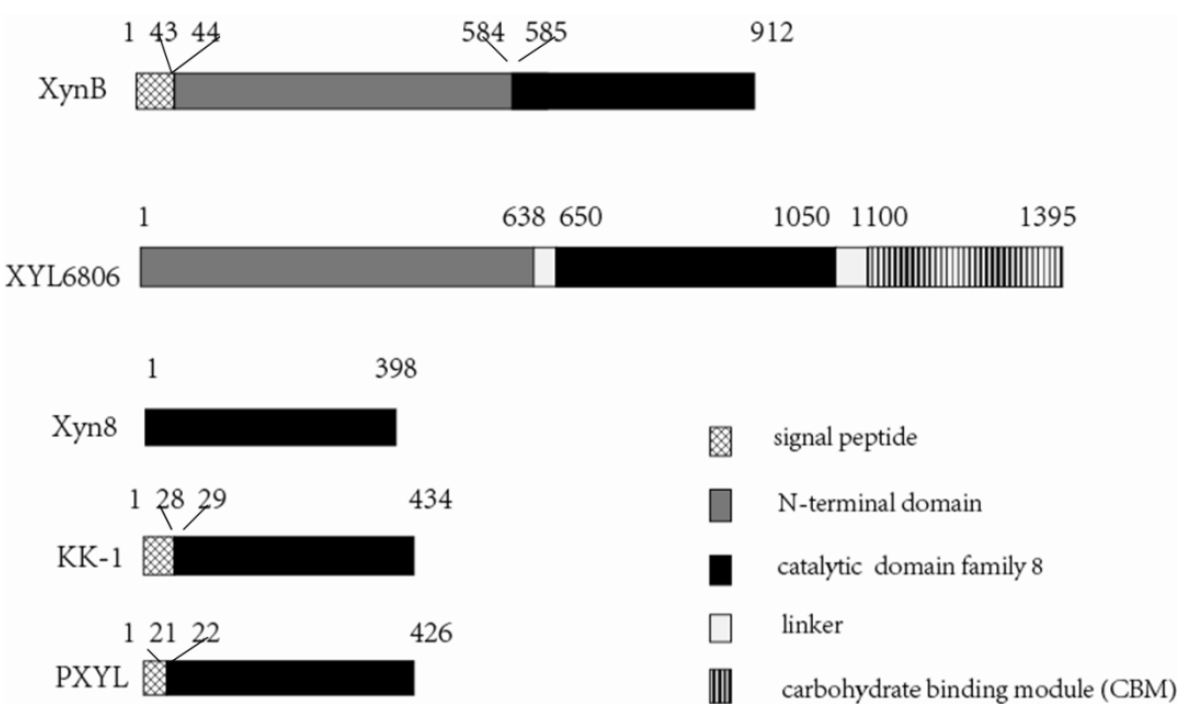

Figure 2. Alignment of catalytic domains of $\mathrm{XynB}$ and some other characterized $\mathrm{GH}$ family 8 xylanases. Stars above residues indicate the conserved catalytic amino acids. Identical residues are shaded in black. XynB, the xylanase in this study (AEC33258); Xyn8, a cold-active xylanase enzyme from an environmental DNA library (ABB71891) [10]; X6806, a xylanase from insect guts (AAS85781) [9]; KK-1, a xylanase from Bacillus sp. KK-1 (AAC27700) [7]; PH, a psychrophilic xylanase from Pseudoalteromonas haloplanktis TAH 3a (AJ427921) [8].

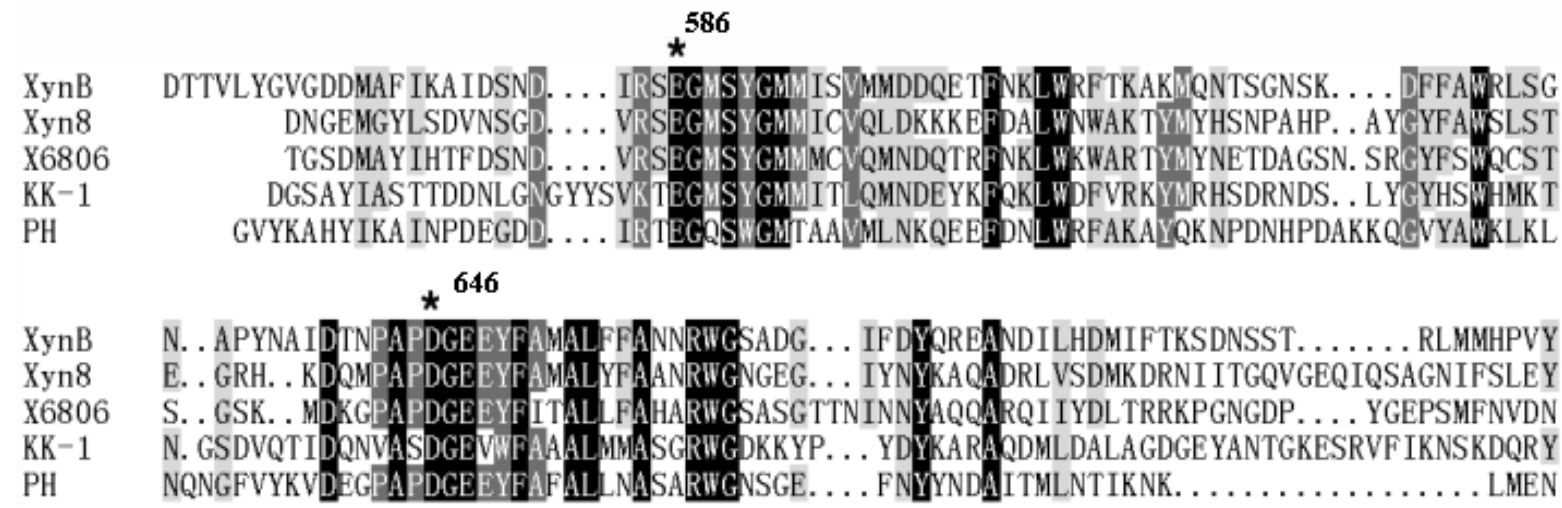


Figure 2. Cont.

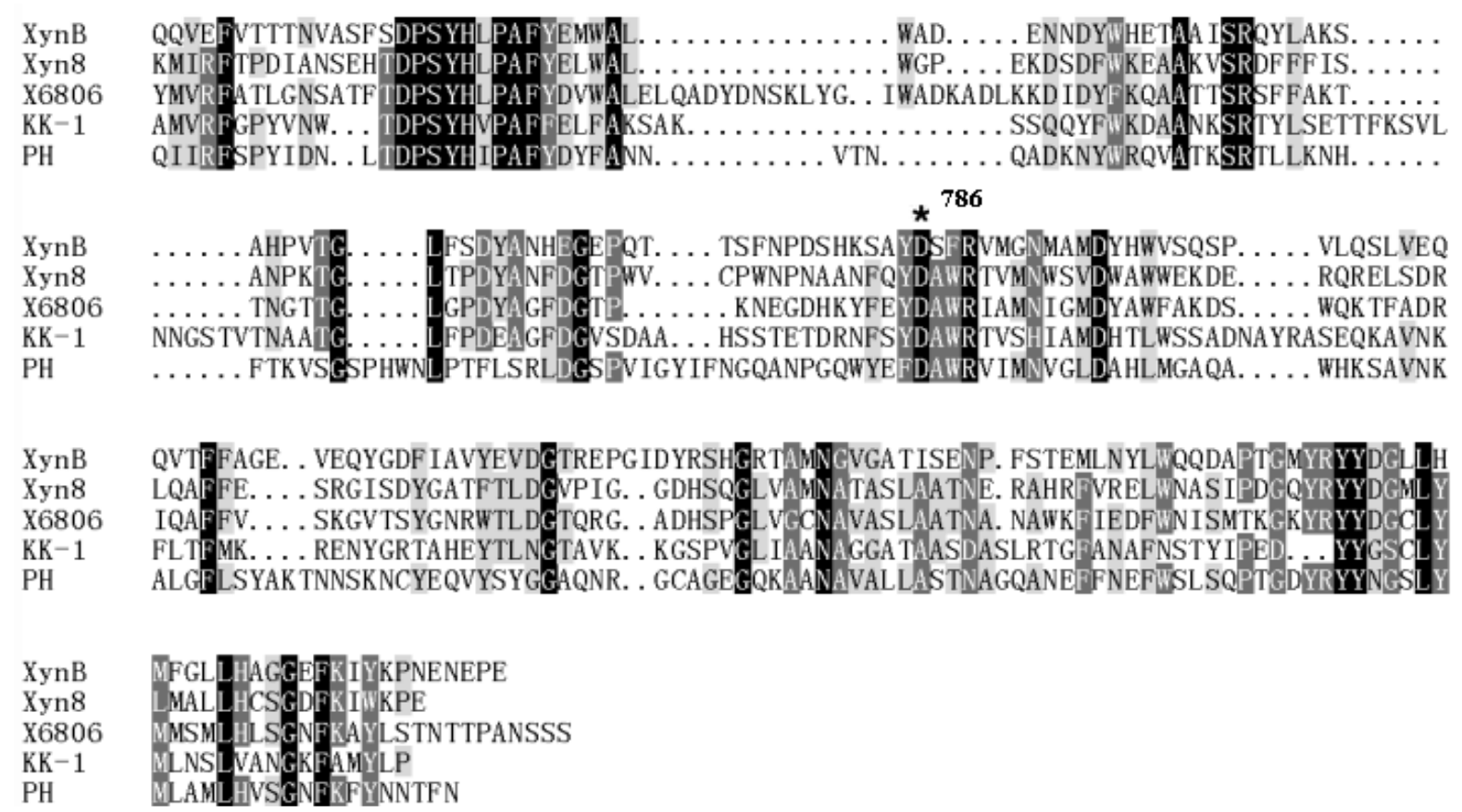

\subsection{Expression and Characterization of $X y n B$}

Gene $x y n B$ was expressed in E. coli, and the recombinant XynB was purified (Figure 3). SDS-PAGE analysis indicated that the recombinant XynB had an apparent $M_{r}$ of about $95 \mathrm{kDa}$ (Figure 3). Since the theoretical molecular mass calculated from the sequence of XynB without the signal peptide is $97.1 \mathrm{kDa}$, the mature XynB should be composed of the NTD and the catalytic domain.

Figure 3. SDS-PAGE analysis of purified recombinant XynB. M, protein molecular weight marker; lanes 1 and 2, proteins in two different tubes after DEAE-Sepharose ion exchange chromatography; lanes 3 and 4, purified XynB in two different tubes after Ni-affinity chromatography.

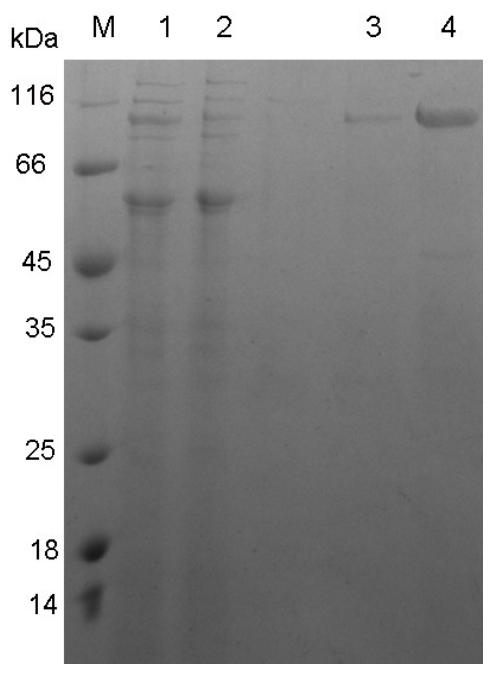

The purified XynB was then characterized. XynB had high activity on natural xylans, such as beech wood xylan and oat spelt xylan, and no detectable activity on cellulose or other glucose-formed 
polysaccharides, such as laminarin, mannan, chitosan and starch, which suggests that XynB is a strict xylanase. Of the two types of xylan tested, XynB showed a higher specific activity toward beech wood xylan (143 $\pm 1.8 \mathrm{U} / \mathrm{mg}$ protein) and a lower specific activity toward soluble oat spelt xylan (108 $\pm 2.3 \mathrm{U} / \mathrm{mg}$ protein) under optimal conditions ( $\mathrm{pH} 7.0$ and $\left.35^{\circ} \mathrm{C}\right)$.

With beech wood xylan as the substrate, $\mathrm{XynB}$ showed the highest activity at $\mathrm{pH}$ 6.0-7.0 (Figure 4A). It was stable between $\mathrm{pH} 6.0$ and 10.0, retaining more than $80 \%$ activity after incubation at $\mathrm{pH}$ 6.0-10.0 for $1 \mathrm{~h}$ (Figure 4B). The optimal temperature for XynB was $35{ }^{\circ} \mathrm{C}$. It retained $7.5 \%$ and $14.6 \%$ activity at $0{ }^{\circ} \mathrm{C}$ and $5{ }^{\circ} \mathrm{C}$, respectively, and had no detectable activity at $60{ }^{\circ} \mathrm{C}$ (Figure $4 \mathrm{C}$ ). As shown in Figure 4D, XynB exhibited very low thermostability, retaining less than $40 \%$ activity after 60 -min incubation at $35{ }^{\circ} \mathrm{C}$ and losing all the activity after 20 -min incubation at $45{ }^{\circ} \mathrm{C}$. These results indicated that $\mathrm{XynB}$ is thermolabile.

Figure 4. $\mathrm{pH}$ and temperature optimums and stabilities of XynB. (A) Effect of $\mathrm{pH}$ on XynB activity. Activities at various $\mathrm{pH}$ were assayed at $35^{\circ} \mathrm{C} ;(\mathbf{B}) \mathrm{pH}$ stability of xynB. Residual activities after incubation at various $\mathrm{pH}$ were assayed at $\mathrm{pH} 7.0$ and $35{ }^{\circ} \mathrm{C}$; (C) Effect of temperature on XynB activity. The assay was performed at $\mathrm{pH} 7.0$ in phosphate buffer; (D) Thermostability of XynB. The enzyme was incubated at $25{ }^{\circ} \mathrm{C}, 35^{\circ} \mathrm{C}$ and $45{ }^{\circ} \mathrm{C}$ for different periods of time, and then, the residual activity was assayed at $35^{\circ} \mathrm{C}$.

A

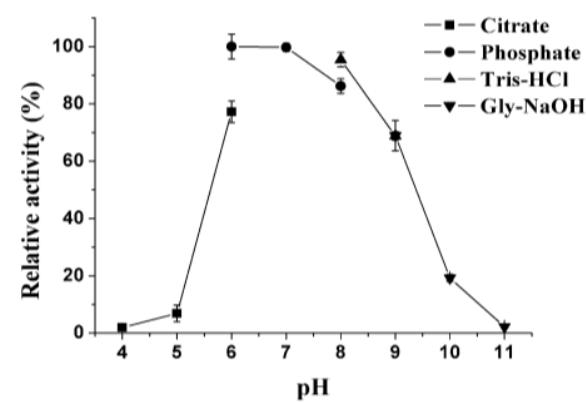

C

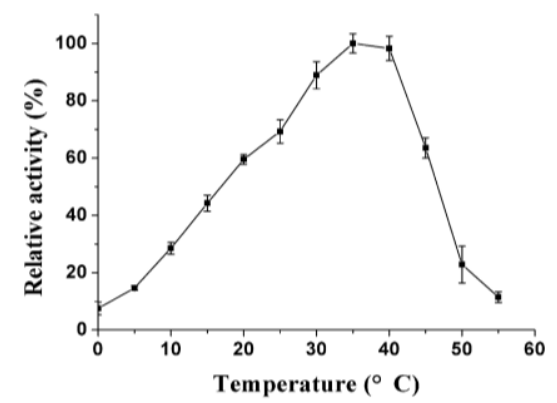

B

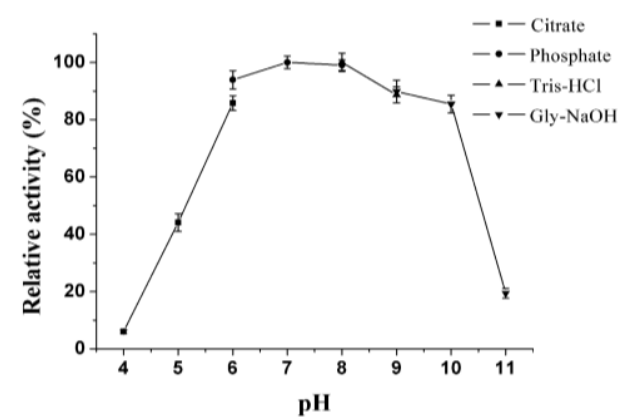

D

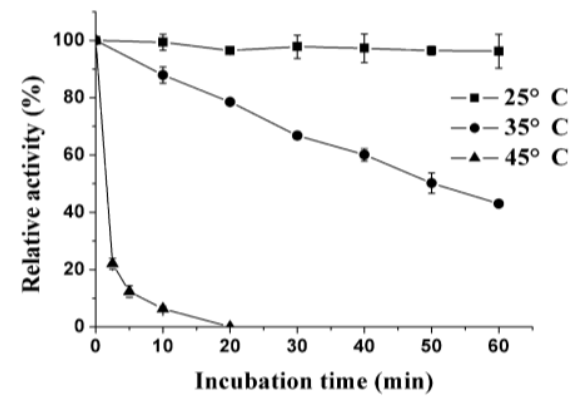

Among all the tested metal ions, $\mathrm{Co}^{2+}(1 \mathrm{mM}$ and $5 \mathrm{mM})$ had the strongest inhibitory effect on the activity, while $\mathrm{Zn}^{2+}, \mathrm{Ni}^{2+}, \mathrm{Mn}^{2+}$ and $\mathrm{Li}^{2+}$ at a concentration of $5 \mathrm{mM}$ had significantly inhibitory effects. No metal ions or chemical reagents showed obvious stimulative effects on the activity (Table 1). The $\mathrm{NaCl}$ concentration of the seawater is about 3\%. Since G. mesophila KMM241 was isolated from a marine invertebrate animal, the effect of $\mathrm{NaCl}$ on $\mathrm{XynB}$ activity was evaluated. $\mathrm{NaCl}$ displayed no evident activation effect on XynB activity. However, XynB showed some salt-tolerant ability, retaining approximately $40 \%$ activity in $4.0 \mathrm{M} \mathrm{NaCl}$ (Figure 5). 
Table 1. Effect of metal ions and chemical reagents on the activity of XynB *.

\begin{tabular}{|c|c|c|}
\hline \multirow{2}{*}{$\begin{array}{c}\text { Metal ions and } \\
\text { chemical reagents }\end{array}$} & \multicolumn{2}{|c|}{ Relative activity (\%) } \\
\hline & $1 \mathrm{mM}$ & $5 \mathrm{mM}$ \\
\hline control & $100 \pm 1.2$ & $100 \pm 0.5$ \\
\hline Urea & $99.2 \pm 3.1$ & $101.5 \pm 2.4$ \\
\hline $\mathrm{Sn}^{2+}$ & $96.3 \pm 0.7$ & $100.4 \pm 1.7$ \\
\hline $\mathrm{Ca}^{2+}$ & $97.9 \pm 0.8$ & $95.6 \pm 1.1$ \\
\hline $\mathrm{K}^{+}$ & $101.3 \pm 2.1$ & $93.7 \pm 0.8$ \\
\hline $\mathrm{Sr}^{2+}$ & $95.7 \pm 1.3$ & $94.5 \pm 2.3$ \\
\hline $\mathrm{Mg}^{2+}$ & $98.7 \pm 0.3$ & $89.7 \pm 1.9$ \\
\hline $\mathrm{Fe}^{3+}$ & $92.5 \pm 0.5$ & $87.7 \pm 3.6$ \\
\hline $\mathrm{Cu}^{2+}$ & $95.7 \pm 1.9$ & $86.4 \pm 2.7$ \\
\hline $\mathrm{Li}^{2+}$ & $94.0 \pm 0.8$ & $76.2 \pm 0.8$ \\
\hline EDTA & $85.8 \pm 2.1$ & $67.6 \pm 1.4$ \\
\hline SDS & $84.9 \pm 1.0$ & $65.0 \pm 1.8$ \\
\hline $\mathrm{Mn}^{2+}$ & $73.4 \pm 2.2$ & $54.2 \pm 0.6$ \\
\hline $\mathrm{Ni}^{2+}$ & $90.7 \pm 3.8$ & $49.7 \pm 0.9$ \\
\hline $\mathrm{Zn}^{2+}$ & $81.1 \pm 0.7$ & $39.0 \pm 2.5$ \\
\hline $\mathrm{Co}^{2+}$ & $64.2 \pm 0.6$ & $8.5 \pm 2.2$ \\
\hline
\end{tabular}

* The activity of $\mathrm{XynB}$ in the presence of $1 \mathrm{mM}$ and $5 \mathrm{mM}$ of each reagent was measured at $\mathrm{pH} 7.0$ and $35{ }^{\circ} \mathrm{C}$, respectively. The activity of $\mathrm{XynB}$ without any reagent was taken as control $(100 \%)$. Each experiment was performed in triplicate.

Figure 5. Effect of $\mathrm{NaCl}$ on the activity of recombinant XynB. The enzyme activity was measured at $35^{\circ} \mathrm{C}$ in phosphate buffer $(\mathrm{pH} 7.0)$ containing $0-4.0 \mathrm{M} \mathrm{NaCl}$.

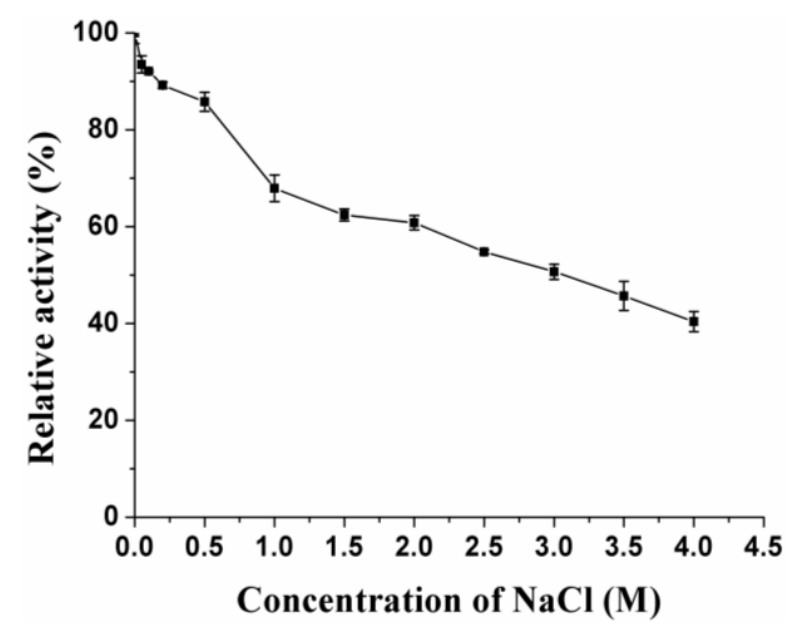

Kinetic parameters of $\mathrm{XynB}$ on beech wood xylan and oat spelt xylan were determined at $35{ }^{\circ} \mathrm{C}$ (Table 2). XynB had lower $K_{\mathrm{m}}$ and higher $k_{\text {cat }} / K_{\mathrm{m}}$ to beech wood xylan than to oat spelt xylan, indicating that XynB had stronger affinity and higher catalytic efficiency to beech wood xylan than to oat spelt xylan. 
Table 2. Kinetic parameters of XynB on two types of xylan *.

\begin{tabular}{ccccc}
\hline Xylan & $\boldsymbol{K}_{\mathrm{m}}(\mathbf{m g} / \mathbf{m L})$ & $\boldsymbol{V}_{\max }(\mathbf{m m o l} / \mathbf{m i n} \cdot \mathbf{m g})$ & $\boldsymbol{k}_{\text {cat }}(\mathbf{1} / \mathbf{s})$ & $\boldsymbol{k}_{\text {cat }} / \boldsymbol{K}_{\mathrm{m}}(\mathbf{m L} / \mathbf{m g} \cdot \mathbf{s})$ \\
\hline Beech wood xylan & 5.82 & 0.38 & 609 & 104.64 \\
Oat spelt xylan & 11.86 & 0.44 & 712 & 60.03 \\
\hline
\end{tabular}

* The $K_{\mathrm{m}}$ and $V_{\max }$ values were determined by Lineweaver-Burk plots, which was made by linear regression, with initial rates determined with $1.0-15.0 \mathrm{~g} / \mathrm{L}$ substrate at $35^{\circ} \mathrm{C}$ using an enzyme concentration ([E]) of $36 \mu \mathrm{g} / \mathrm{mL}$. $k_{\text {cat }}$ values were calculated with the formula $k_{\text {cat }}=V_{\max } /[\mathrm{E}]$.

\subsection{Hydrolysis Product Analysis}

Xylo-oligosaccharides, $\mathrm{x} 3-\mathrm{x} 6$, were hydrolyzed by $\mathrm{XynB}$ at $15^{\circ} \mathrm{C}$ for $12 \mathrm{~h}$, respectively, and the products were analyzed using HPLC. As shown in Figure 6, XynB could not hydrolyze xylotetraose and xylotriose and could hydrolyze xylohexaose and xylopentaose. Xylohexaose was completely hydrolyzed, producing xylobiose, xylotriose and xylotetraose. The hydrolysis efficiency to xylopentaose was relatively low, producing a small amount of xylotriose and xylobiose. No xylose was detected in all hydrolysis process. These results showed that XynB is a strict endo- $\beta-1,4-x y l a n a s e$ exhibiting an action pattern with a demand of at least five sugar moieties for effective cleavage.

Figure 6. HPLC analysis of the products of $x y l o$-oligosaccharides hydrolyzed by XynB. S, a mixture of $\mathrm{x} 1-\mathrm{x} 6 ; 1$, hydrolysis products of xylohexaose; 2 , hydrolysis products of xylopentaose; 3 , hydrolysis products of xylotetraose; 4 , hydrolysis products of xylotriose.

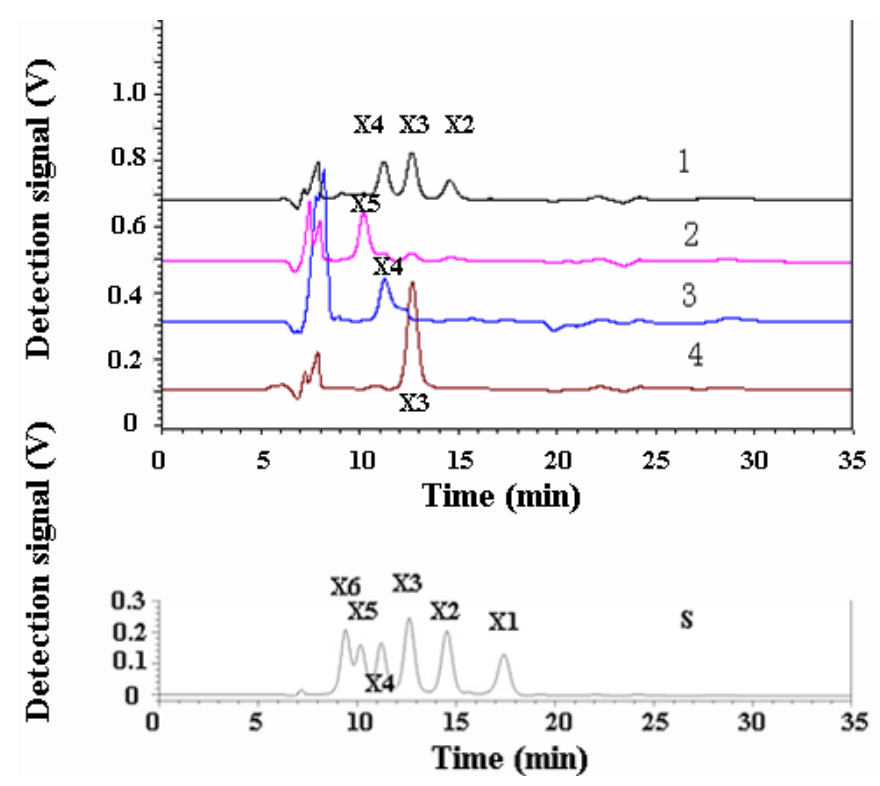

\subsection{Insoluble Polysaccharide-Binding Ability of the N-Terminal Domain}

Some xylanases own one or several CBMs. In order to investigate whether the NTD of XynB functions as a CBM, we expressed NTD and examined the binding ability of the recombinant NTD to four insoluble polysaccharides by SDS-PAGE. As shown in Figure 7, the recombinant NTD exhibited strong binding ability to insoluble oat spelt xylan and avicel, but little binding ability to chitosan and chitin. This result suggested that the NTD of XynB may function as a substrate-binding domain during xylan hydrolysis. CBMs are now classified into 66 families on the basis of amino acid sequence 
similarities in the CAZy database. Blast analysis indicated that the NTD of XynB has no obvious homology to any CBM sequence in public databases. Therefore, the NTD of XynB may contain a new type of CBM. In this experiment, BSA was used as a negative control.

Figure 7. SDS-PAGE analysis of binding of the recombinant $N$-terminal domain (NTD) to different insoluble polysaccharides. Bovine serum albumin (BSA) $(0.1 \mathrm{mg})$ was used as a negative control. Proteins were mixed with the insoluble polysaccharides for $1 \mathrm{~h}$; bound and unbound fractions were separated by centrifugation and analyzed by $12.5 \%$ SDS-PAGE. The proteins in the gels were visualized by staining with $0.1 \%$ Coomassie blue R 250 . Lane C, control protein; lane 1, unbound fraction; lane 2, protein in the wash buffer; lane 3, fraction adsorbed to the polymer.

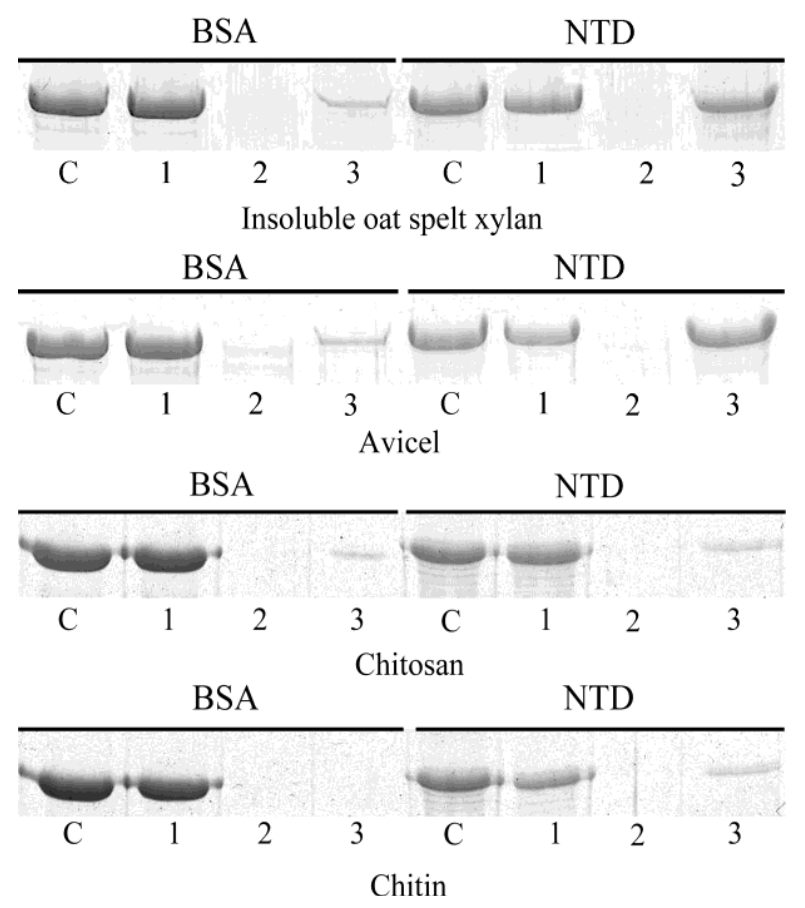

\section{Discussion}

Endo- $\beta$-1,4-xylanases (EC 3.2.1.8) play a crucial role in xylan degradation and, thus, have important applications in industries, such as textile, paper, food, animal feed, etc. Therefore, searching for novel xylanases with better properties for specific applications from different environments is a long-lasting interest, although xylanases have been extensively studied for many years. In this study, a xylanase gene, $x y n B$, was cloned from the marine bacterium, G. mesophila KMM241, and expressed in E. coli. The recombinant $\mathrm{XynB}$ is a GH family 8 multi-domain endo- $\beta$-1,4-xylanase containing an NTD with unknown function and a catalytic domain of GH family 8. Up to now, only four endo- $\beta-1,4$-xylanases belonging to $\mathrm{GH}$ family 8 have been characterized. They are XynY from Bacillus sp. KK-1 [7], PhXyl from P. haloplanktis [8], XYL6806 from an insect gut microbe [9] and rXyn8 from an environmental DNA library [10]. Among these GH family 8 xylanases, XynB has the highest identity (38\%) to the recombinant xylanase rXyn8. In the four GH family 8 xylanases, except XYL6806, which is a modular enzyme containing an NTD with an unknown function, a catalytic domain and a CBM, the other three are mono-domain enzymes containing only a catalytic domain 
(Figure 1). Blast analysis showed that the NTD of XynB has no obvious homology to any sequence in public databases. Our sequence alignment showed that the homology between the NTD of XynB and that of XYL6806 is only $13.44 \%$. Therefore, XynB is a structurally novel xylanase of GH family 8 .

The recombinant $\mathrm{XynB}$ has a $\mathrm{pH}$ optimum of 6-7 and a temperature optimum of $35^{\circ} \mathrm{C}$. The $K_{\mathrm{m}}$ and $k_{\text {cat }}$ value of $\mathrm{XynB}$ is $5.82 \mathrm{mg} / \mathrm{mL}$ and $609 / \mathrm{s}$, comparative to those of $\mathrm{rXyn} 8(5.3 \mathrm{mg} / \mathrm{mL}$ and 588/s) [10] and lower than that of PhXyl (28 mg/mL and 1247/s) [8]. XynB was thermolabile and salt-tolerant, probably because it was from a marine bacteria isolated from an invertebrate from the Arctic sea [16]. XynB is the first reported salt-tolerant xylanase in GH family 8, which is comparable to some other marine xylanases $[14,15,25]$. In previous reports, $\mathrm{Cu}^{2+}$ seems to strongly inhibit the activity of several xylanases [26-28]. Therefore, $\mathrm{Cu}^{2+}$ is considered as one of the main adverse factors for industrial application of xylanase [29]. However, our study showed that $\mathrm{Cu}^{2+}$ had no evident effect on XynB activity, while $\mathrm{Co}^{2+}, \mathrm{Zn}^{2+}, \mathrm{Ni}^{2+}, \mathrm{Mn}^{2+}$ and $\mathrm{Li}^{2+}$ had an inhibitory effect. This result is in accordance with the other GH family 10 xylanase, XynA, from G. mesophila KMM241 [14], but different from $\mathrm{PhXyl}$, which is inhibited by heavy metals, such as $\mathrm{Hg}^{2+}, \mathrm{Cu}^{2+}, \mathrm{Zn}^{2+}$ and $\mathrm{Ni}^{2+}$ [8].

Substrate specificity analysis showed that the recombinant XynB was a strict endo-xylanase and did not have any $\beta$-glucosidase activity, which is in accordance with all the other reported family 8 endo-xylanases. However, the hydrolysis product profiles of different family 8 endo-xylanases seem to be quite different. XynB seems to function in a similar way to XYL6806 and PhXyl. All of these three enzymes did not hydrolyze xylotriose, and activity on xylotetraose was too low to detect. They hydrolyzed xylopentaose into xylobiose and xylotriose in a low catalytic efficiency, but efficiently hydrolyzed xylohexaose into xylobiose, xylotriose and xylotetraose [8,9]. rXyn8 functions in a different way, selectively releasing xylotriose from substrates [10]. Structural comparison of rXyn8 and $\mathrm{PhXyl}$ showed that subtle amino acid changes in the glycon, as well as the aglycon sub-sites probably form the basis of the observed differences between GH family 8 xylanases [6].

Xylanases have potential applications in a wide range of industrial processes, such as food processing, animal feeds, paper and pulp, textiles and bioremediation, etc. [2]. As a strict endo-xylanase, XynB may have a potential in some of these industrial processes. Especially, XynB is thermolabile, which may have advantages in the food industries, where a high temperature for enzyme inactivation is not allowed. As a salt-tolerant enzyme, xynB may have a potential in biotechnological processes where the catalysis environment is highly salty, such as in the processing of sea food and saline food.

Many glycoside hydrolases, especially those utilizing insoluble matter as substrates, are modular enzymes with two or more modules, including catalytic modules and carbohydrate-binding modules (CBMs). CBMs are now classified into 66 families on the basis of amino acid sequence similarities in the CAZy database. It is generally considered that CBMs facilitate the access of enzymes to polysaccharides and promote polysaccharides degradation [30,31]. Some xylanases own one or several CBMs. For example, a thermostable multi-domain 1,4- $\beta$-xylanase from Caldibacillus cellulovorans has a $\mathrm{CBM}$ at its $N$-terminus [32]. A multi-domain endo- $\beta-1,4$-xylanase from Paenibacillus curdlanolyticus B-6 has three family $22 \mathrm{CBMs}$ at its $N$-terminus and one family $9 \mathrm{CBM}$ at its $C$-terminus [33]. Among the characterized family 8 endo-xylanases, only XYL6806 is a modular enzyme containing a family $3 \mathrm{CBM}$ at its $C$-terminus [9]. In this study, the NTD of XynB was found to have insoluble polysaccharide-binding ability, indicating that it may contain a CBM. As the amino acid sequence of the NTD of XynB has no obvious homology to any CBM sequence in public 
databases, XynB may contain a new type of CBM, of which the structure and function need to be further elucidated.

\section{Conclusion}

Gene $x y n B$ cloned from the marine bacterium, Glaciecola mesophila KMM241, encodes a multi-domain xylanase XynB of GH family 8, which has a low identity (38\%) to the characterized xylanases. The recombinant XynB with an apparent $M_{r}$ of about $95 \mathrm{kDa}$ contains an NTD with an unknown function and a catalytic domain. The recombinant XynB showed optimal activity at $\mathrm{pH}$ 6-7 and $35{ }^{\circ} \mathrm{C}$. XynB is an endo-xylanase hydrolyzing xylo-oligosaccharide with at least five sugar moieties into xylotetraose, xylotriose and xylobiose. XynB was thermolabile and salt-tolerant, which may endue it an advantage in some biotechnological processes. The recombinant NTD exhibited high binding ability to insoluble polysaccharides, such as xylan and avicel. The NTD shows no obvious homology to any other $\mathrm{CBM}$ sequence in public databases, suggesting that XynB may contain a new type of CBM. These results indicate that XynB is a novel xylanase of GH family 8.

\section{Acknowledgments}

The work was supported by the National Natural Science Foundation of China (41176130, 41106161, 31025001, 31070061, 31000034), the Hi-Tech Research and Development program of China (2011AA09070303, 2011AA090704, 2012AA092105, 2012AA092103), the China Ocean Mineral Resources R \& D Association (COMRA) Special Foundation (DY125-15-T-05), Natural Science Foundation of Shandong Province, China (JQ200910), the Foundation for Young Excel-lent Scientists in Shandong Province (BS2010SW015) and the Independent Innovation Foundation of Shandong University (2009TS079, 2012TB004).

\section{References}

1. Beg, Q.K.; Kapoor, M.; Mahajan, L.; Hoondal, G.S. Microbial xylanases and their industrial applications: A review. Appl. Microbiol. Biotechnol. 2001, 56, 326-338.

2. Collins, T.; Gerday, C.; Feller, G. Xylanases, xylanase families and extremophilic xylanases. FEMS Microbiol. Rev. 2005, 29, 3-23.

3. Saha, B.C.; Bothast, R.J. Enzymology of Xylan Degradation. In Biopolymers; Imam, S.H., Greene, R.V., Zaidi, B.R., Eds.; American Chemical Society: Washington, DC, USA, 1999; pp. 167-194.

4. Dhiman, S.S.; Sharma, J.; Battana, B. Industrial applications and future prospects of microbial xylanases: A review. BioResources 2008, 3, 1377-1402.

5. Juturu, V.; Wu, J.C. Microbial xylanases: Engineering, production and industrial applications. Biotechnol. Adv. 2011, 30, 1219-1227.

6. Pollet, A.; Schoepe, J.; Dornez, E.; Strelkov, S.V.; Delcour, J.A.; Courtin, C.M. Functional analysis of glycoside hydrolase family 8 xylanases shows narrow but distinct substrate specificities and biotechnological potential. Appl. Microbiol. Biotechnol. 2010, 87, 2125-2135. 
7. Yoon, K.H.; Yun, H.N.; Jung, K.H. Molecular cloning of a Bacillus sp. KK-1 xylanase gene and characterization of the gene product. Biochem. Mol. Biol. Int. 1998, 45, 337-347.

8. Collins, T.; Meuwis, M.A.; Stals, I.; Claeyssens, M.; Feller, G.; Gerday, C. A novel family 8 xylanase: Functional and physicochemical characterization. J. Biol. Chem. 2002, 277, 35133-35139.

9. Brennan, Y.; Callen, W.N.; Christoffersen, L.; Dupree, P.; Goubet, F.; Healey, S.; Hernandez, M.; Keller, M.; Li, K.; Palackal, N.; et al. Unusual microbial xylanases from insect guts. Appl. Environ. Microbiol. 2004, 70, 3609-3617.

10. Lee, C.C.; Kibblewhite-Accinelli, R.E.; Wagschal, K.; Robertson, G.H.; Wong, D.W. Cloning and characterization of a cold-active xylanase enzyme from an environmental DNA library. Extremophiles 2006, 10, 295-300.

11. Honda, Y.; Kitaoka, M. A family 8 glycoside hydrolase from Bacillus halodurans C-125 (BH2105) is a reducing end xylose-releasing exo-oligoxylanase. J. Biol. Chem. 2004, 279, 55097-55103.

12. Van den Broek, L.A.M.; Lloyd, R.M.; Beldman, G.; Verdoes, J.C.; McCleary, B.V.; Voragen, A.G.J. Cloning and characterization of arabinofuranohydrolase-D3 (AXHd3) from Bifidobacterium adolescentis DSM20083. Appl. Microbiol. Biotechnol. 2005, 67, 641-647.

13. Wu, S.; Liu, B.; Zhang, X. Characterization of a recombinant thermostable xylanase from deep-sea thermophilic Geobacillus sp. MT-1 in East Pacific. Appl. Microbiol. Biotechnol. 2006, $72,1210-1216$.

14. Guo, B.; Chen, X.L.; Sun, C.Y.; Zhou, B.C.; Zhang, Y.Z. Gene cloning, expression and characterization of a new cold-active and salt-tolerant endo- $\beta-1,4$-xylanase from marine Glaciecola mesophila KMM241. Appl. Microbiol. Biotechnol. 2009, 84, 1107-1115.

15. Liu, Z.; Zhao, X.Q.; Bai, F.W. Production of xylanase by an alkaline-tolerant marine-derived Streptomyces viridochromogenes strain and improvement by ribosome engineering. Appl. Microbiol. Biotechnol. 2012, doi:10.1007/s00253-012-4290-y.

16. Romanenko, L.A.; Zhukova, N.V.; Rohde, M.; Lysenko, A.M.; Mikhailov, V.V.; Stackebrandt, E. Glaciecola mesophila sp. nov., a novel marine agar-digesting bacterium. Int. J. Syst. Evol. Microbiol. 2003, 53, 647-651.

17. Zheng, H.; Guo, B.; Chen, X.L.; Fan, S.J.; Zhang, Y.Z. Improvement of the quality of wheat bread by addition of glycoside hydrolase family 10 xylanases. Appl. Microbiol. Biotechnol. 2011, 90, 509-515.

18. Saito, H.; Miura, K. Preparation of transforming deoxyribonucleic acid by phenol treatment. Biochim. Biophys. Acta 1963, 72, 619-629.

19. Laemmli, U.K. Cleavage of structural proteins during the assembly of the head of bacteriophage T4. Nature 1970, 227, 680-685.

20. Miller, G.L.; Blum, R.; Glennon, W.E.; Burton, A.L. Measurement of carboxymethyl cellulase activity. Anal. Biochem. 1960, 2, 127-132.

21. Bradford, M.M. A rapid and sensitive method for the quantitation of microgram quantities of protein utilizing the principle of protein-dye binding. Anal. Biochem. 1976, 72, 248-254.

22. Pason, P.; Kyu, K.L.; Ratanakhanokchai, K. Paenibacillus curdlanolyticus strain B-6 xylanolytic-cellulolytic enzyme system that degrades insoluble polysaccharides. Appl. Environ. Microbiol. 2006, 72, 2483-2490. 
23. Valenzuela, S.V.; Diaz, P.; Pastor, F.L.J. Modular glucuronoxylan-specific xylanase with a family CBM35 carbohydrate-binding module. Appl. Environ. Microbiol. 2012, 78, 3923-3931.

24. Cantarel, B.C.; Coutinho, P.M.; Rancurel, C.; Bernard, T.; Lombard, T.; Henrissat, B. The Carbohydrate-Active EnZymes database (CAZy): An expert resource for Glycogenomics. Nucleic Acids Res. 2009, 37, D233-D238.

25. Hung, K.S.; Liu, S.M.; Tzou, W.S.; Lin, F.P.; Pan, C.L.; Fang, T.Y.; Sun, K.H.; Tang, S.J. Characterization of a novel GH10 thermostable, halophilic xylanase from the marine bacterium Thermoanaerobacterium saccharolyticum NTOU1. Proc. Biochem. 2011, 46, 1257-1263.

26. Fialho, M.B.; Carmona, E.C. Purification and characterization of xylanase from Aspergillus giganteus. Folia Microbiol. 2004, 49, 13-18.

27. Gupta, S.; Bhushan, B.; Hoondal, G.S. Isolation, purification and characterization of xylanase from Staphylococcus sp. SG-13 and its application in biobleaching of kraft pulp. J. Appl. Microbiol. 2000, 88, 325-334.

28. Yamaura, I.; Koga, T.; Matsumoto, T.; Kato, T. Purification and some properties of endo-1,4- $\beta$-D-xylanase from a fresh-water mollusc, Pomacea insularus (de Ordigny). Biosci. Biotechnol. Biochem. 1997, 61, 615-620.

29. Zhang, G.; Huang, J.; Huang, G.; Ma, L.; Zhang, X. Molecular cloning and heterologous expression of a new xylanase gene from Plectosphaerella cucumerina. Appl. Microbiol. Biotechnol. 2007, 74, 339-346.

30. Ali, M.K.; Hayashi, H.; Karita, S.; Goto, M.; Kimura, T.; Sakka, K.; Ohmiya, K. Importance of the carbohydrate-binding module of Clostridium stercorarium Xyn10B to xylan hydrolysis. Biosci. Biotechnol. Biochem. 2001, 65, 41-47.

31. Carrard, G.; Koivula, A.; Soderlund, H.; Beguin, P. Cellulose-binding domains promote hydrolysis of different sites on crystalline cellulose. Proc. Natl. Acad. Sci. USA 2000, 97, $10342-10347$.

32. Sunna, A.; Gibbs, M.D.; Bergquist, P.L. A novel thermostable multidomain 1,4- $\beta$-xylanase from 'Caldibacillus cellulovorans' and effect of its xylan-binding domain on enzyme activity. Microbiology 2000, 146, 2947-2955.

33. Waeonukul, R.; Pason, P.; Kyu, K.L.; Sakka, K.; Kosugi, A.; Mori, Y.; Ratanakhanokchai, K. Cloning, sequencing, and expression of the gene encoding a multidomain endo- $\beta-1,4-x y l a n a s e$ from Paenibacillus curdlanolyticus B-6, and characterization of the recombinant enzyme. J. Microbiol. Biotechnol. 2009, 19, 277-285.

(C) 2013 by the authors; licensee MDPI, Basel, Switzerland. This article is an open access article distributed under the terms and conditions of the Creative Commons Attribution license (http://creativecommons.org/licenses/by/3.0/). 